\title{
Continuous Protection of a Collective State from Inhomogeneous Dephasing
}

\author{
R. Finkelstein $\odot,{ }^{1}$ O. Lahad, ${ }^{1}$ I. Cohen $\odot,{ }^{2}$ O. Davidson, ${ }^{1}$ S. Kiriati, ${ }^{1}$ E. Poem $\odot,{ }^{1}$ and O. Firstenberg ${ }^{1}$ \\ ${ }^{1}$ Physics of Complex Systems, Weizmann Institute of Science, Rehovot 7610001, Israel \\ ${ }^{2}$ Department of Physics and Astronomy, Aarhus University, \\ Ny Munkegade 120, DK-8000 Aarhus C, Denmark
}

(Received 23 April 2020; revised 1 October 2020; accepted 23 November 2020; published 13 January 2021)

\begin{abstract}
We introduce and demonstrate a scheme for eliminating the inhomogeneous dephasing of a collective quantum state. The scheme employs off-resonant fields that continuously dress the collective state with an auxiliary sensor state, which has an enhanced and opposite sensitivity to the same source of inhomogeneity. We derive the optimal conditions under which the dressed state is fully protected from dephasing when using either one or two dressing fields. The latter provides better protection, circumvents qubit phase rotation, and suppresses the sensitivity to drive noise. We further derive expressions for all residual, higherorder sensitivities. We experimentally study the scheme by protecting a collective excitation of an atomic ensemble, where inhomogeneous dephasing originates from thermal motion. Using photon storage and retrieval, we demonstrate complete suppression of inhomogeneous dephasing and, consequently, a prolonged memory time. Our scheme may be applied to eliminate motional dephasing in other systems, improving the performance of quantum gates and memories with neutral atoms. It is also generally applicable to various gas, solid, and engineered systems, where sensitivity to variations in time, space, or other domains limits possible scale-up of the system.
\end{abstract}

DOI: 10.1103/PhysRevX.11.011008

Subject Areas: Atomic and Molecular Physics,

Quantum Information

\section{INTRODUCTION}

The quantum state of a system is prone to decoherence via inhomogeneous dephasing due to variations among the system's constituents. These variations include spatial inhomogeneities, primarily nonuniform external fields [1-3], environmental imperfections [4,5], finite temperature effects such as thermal velocity distribution [6-10], and fabrication infidelities in engineered systems [11-13]. Slow temporal fluctuations and shot-to-shot variations, also relevant to single-constituent systems, may also manifest as inhomogeneous dephasing [9,14-16]. In quantum information processing, these variations limit the qubit coherence time.

In some cases, such as in dual-color magic-wavelength optical traps, it is possible to minimize inhomogeneous dephasing by introducing an additional field that induces exactly the same inhomogeneity and thus directly balances the differential phase shifts [17-21]. In more general cases, inhomogeneous dephasing can be mitigated by pulse-based protocols, namely, echo sequences and various dynamical decoupling methods [22-25]. In these protocols, the system

Published by the American Physical Society under the terms of the Creative Commons Attribution 4.0 International license. Further distribution of this work must maintain attribution to the author(s) and the published article's title, journal citation, and DOI. regains coherence only at discrete, predetermined times. Several protocols of continuous dynamical decoupling have been studied [16,26-28], typically relying on strong resonant driving of the qubit transition. Such driving is not compatible with all systems and requires additional measures for eliminating the sensitivity to drive noise.

Here, we propose and demonstrate an alternative approach for mitigating inhomogeneous dephasing. Our scheme employs off-resonance fields for continuously dressing the target state with an additional sensor state, which has an opposite and enhanced sensitivity to the same source of inhomogeneity. This admixture of a portion of the sensor state forms a protected, dephasing-free state. Notably, the dressing field is a continuous wave, providing continuous protection and not limiting the extraction of quantum information to predefined times.

Experimentally, we focus on optical excitations in thermal atomic ensembles. Storing and processing quantum information as collective excitations in atomic ensembles offers long coherence times [29-33], noiseless memory protocols [7,34], remote entanglement [35-37], and the generation of nonclassical light [38,39]. Nevertheless, whether they are warm or ultracold, the atoms are not stationary; their inhomogeneous velocity distribution leads to dephasing of collective states formed by excitations with nonzero momentum transfer $[9,10,40]$. Such motional dephasing is a prevailing decoherence mechanism, yet it is rarely mitigated by means beyond actual cooling and 
trapping of the atoms. While some pulsed spin-echo techniques have been proposed [41] and demonstrated [25], a robust and continuous protocol for protection from motional dephasing has not been realized to date.

In the following, we first present a general analysis of the protection scheme with either one or two dressing fields. We identify the minimal requirements and conditions for optimal protection. We show that introducing two dressing fields reduces not only the sensitivity to frequency variations but also to fluctuations in the dressing fields, known as drive noise $[27,42,43]$. We then turn to the case study of motional dephasing in atomic ensembles. Our experimental realization is based on light storage in a fast ladder memory (FLAME) [7], using the retrieval efficiency as a measure of atomic coherence [44]. We demonstrate complete cancellation of motional dephasing, prolonging the memory lifetime, and we verify the scaling of optimal conditions for protection. We further confirm that the double-dressing scheme, in contrast to the single-dressing scheme, inflicts no qubit phase rotation and is thus less sensitive to drive noise, providing robust continuous protection.

\section{CONTINUOUS PROTECTION USING A SENSOR STATE}

Consider a qubit comprising states $|\downarrow\rangle$ and $|\uparrow\rangle$, whose transition frequency experiences an inhomogeneous shift $\delta$ [Fig. 1(a)]. We require that one of these states-here, chosen to be $|\uparrow\rangle$, without loss of generality-can be coupled by an external dressing field to a third state $|S\rangle$, which acts as a sensor. We further require that the overall transition frequency between $|\downarrow\rangle$ and $|S\rangle$ be sensitive to the same source of inhomogeneity, such that it experiences an opposite and possibly larger shift, $-s \delta$, where $s$ is the sensitivity factor. For example, in the case of motional dephasing, $s$ is the ratio between the Doppler widths of the transitions $|\downarrow\rangle-|S\rangle$ and $|\downarrow\rangle-|\uparrow\rangle$, and, in the case of magnetically sensitive transitions, $s$ is the ratio between their magnetic moment differences. If the above requirements are satisfied, the dressing field can be tuned to protect the qubit from inhomogeneous dephasing.

We begin by considering a single dressing field with Rabi frequency $\Omega$ and detuning $\Delta$ [Fig. 1(b)]. The states $|\uparrow\rangle$ and $|S\rangle$ are mixed, forming dressed states. With respect to $|\downarrow\rangle$, the states $|S\rangle$ and $|\uparrow\rangle$ are inhomogeneously shifted by $[\Delta+\delta(s-1)] / 2 \pm \sqrt{[\Delta+(s+1) \delta]^{2} / 4+\Omega^{2}}$. Optimal protection is achieved when the transition frequency of the qubit $(|\downarrow\rangle-|\uparrow\rangle)$ is insensitive to variations in $\delta$ to first order, which occurs when

$$
\Omega^{2} / \Delta^{2}=s /(s-1)^{2} .
$$

This condition is shown in Fig. 1(d) by the orange line. Since the ratio $\Omega^{2} / \Delta^{2}$ determines the magnitude of mixing between the bare states, the larger the sensitivity factor $s$,

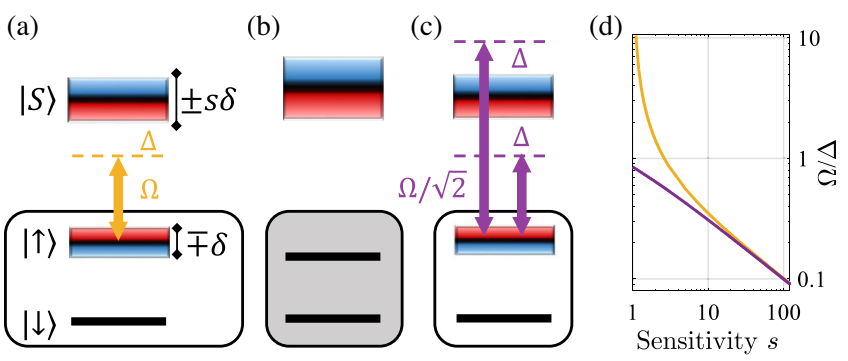

FIG. 1. Continuous protection of a quantum state. (a) Transition frequency of the qubit $|\downarrow\rangle-|\uparrow\rangle$, without protection, shifted by $\delta$ due to some source of inhomogeneity, and an auxiliary sensor state $|S\rangle$, which experiences an opposite and possibly larger shift, $-s \delta$. A single field with Rabi frequency $\Omega$ and detuning $\Delta$ dresses the qubit with the sensor state. (b) Protected qubit formed under conditions of optimal dressing. The transition frequency of the protected qubit is slightly altered by the mean light shift. (c) Two dressing fields of equal intensities and opposite detunings, applied to further eliminate the mean shift and reduce the sensitivity of the protected qubit to dressing noise. (d) Optimal dressing ratio $\Omega / \Delta$ as a function of the sensitivity parameter $s$ for the single-dressing (orange) and double-dressing (purple) schemes.

the smaller the portion of $|S\rangle$ admixed into $|\uparrow\rangle$ at optimal protection.

For $s \gg 1$, the dressed-state frequency shift takes the form of a light shift: $\Omega^{2} /(\Delta+s \delta) \approx\left(\Omega^{2} / \Delta\right)[1-s \delta / \Delta+$ $\left.O(s \delta / \Delta)^{2}\right]$. It is the first-order term $(\Omega / \Delta)^{2} s \delta$ that counteracts the inhomogeneous shift $\delta$ when condition (1) is fulfilled. Given a distribution of inhomogeneous shifts $\delta$ of width $\sigma$, a dressing-field detuning $\Delta \gg s \sigma$ allows efficient protection for practically all $\delta$. This regime has recently been used for narrowing and enhancement of spectral lines $[6,20]$.

A caveat of the single-dressing scheme is the mean, zeroth-order shift $\Omega^{2} / \Delta$ added to the transition frequency of the protected qubit. This shift rotates the qubit phase in time, exposing it to noise in the drive field $\Omega$. To avoid this shift, two dressing fields at opposite detunings $\pm \Delta$ should be used [Fig. 1(c)]. The mean shifts they induce are opposite in sign and thus cancel. In fact, as all even-order terms cancel, a protection up to third order in $\delta / \Delta$ is achieved.

The above arguments apply to any $s$. For a general solution with more than one dressing field, we use the Magnus expansion of the time-dependent Hamiltonian and find the condition under which the transition frequency is insensitive to variations in $\delta$ to first and second orders:

$$
J_{0}(2 \sqrt{2} \Omega / \Delta)=(s-1) /(s+1),
$$

where $J_{0}$ is the zeroth-order Bessel function (see Appendix B for a detailed derivation). Equation (2) converges to Eq. (1) for $s \gg 1$, but $\Omega / \Delta$ scales more favorably with $s$ for small $s$, such that less Rabi frequency and less mixing are required with double dressing [see Fig. 1(d)]. 


\section{MOTIONAL DEPHASING}

Initializing an ensemble of atoms in a collective state is typically done by optical excitation. When the wave vector $k=2 \pi / \Lambda$ of the collective excitation is nonzero, a spatial phase $e^{i(2 \pi / \Lambda) z}$ is imprinted on the atoms, as illustrated in Fig. 2(a). In multiphoton transitions, the excitation wave vector is the vectorial sum of the participating fields' wave vectors. For a single excitation, the collective state can be written as a spin wave $|W\rangle=$ $(1 / \sqrt{N}) \sum_{j} e^{i(2 \pi / \Lambda) z_{j}}|\uparrow\rangle_{j}\left\langle\left.\downarrow\right|_{j} \mid G\right\rangle$, where $z_{j}$ is the position of atom $j$ at storage time $t=0$, and $|G\rangle=\prod_{j}|\downarrow\rangle_{j}$ is the initial collective ground state. When the atoms are displaced by thermal motion, atoms with different thermal velocities $v_{j}$ carry the original phase to different positions $z_{j}+v_{j} t$ along the spin wave [Fig. 2(b)]. The resulting state is

$$
\left|W^{\prime}(t)\right\rangle=\sum_{j} e^{i(2 \pi / \Lambda)\left(z_{j}-v_{j} t\right)}|\uparrow\rangle_{j}\left\langle\left.\downarrow\right|_{j} \mid G\right\rangle,
$$

whose overlap with $|W\rangle$, quantified by the squared coherence $\mathcal{C}(t) \equiv\left|\left\langle W \mid W^{\prime}(t)\right\rangle\right|^{2}$, determines the retrieval

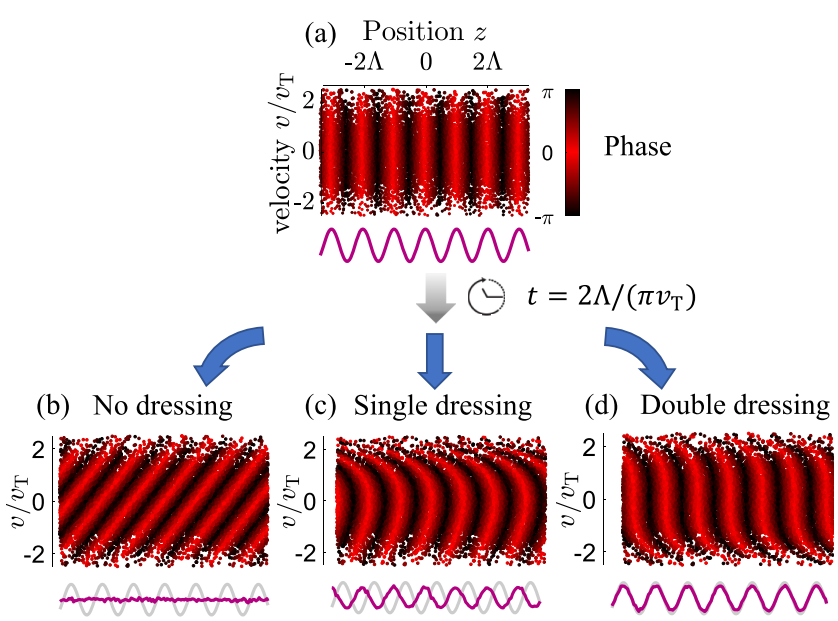

FIG. 2. Motional dephasing and protection of a spin wave. (a) Position-velocity representation of a collective excitation (spin wave), generated, e.g., by light storage in a two-photon bichromatic transition. The collective state has a spatially dependent phase with a wavelength $\Lambda$ (top), clearly apparent when the real part of the spin wave is averaged over all velocities (purple, bottom). (b) Thermal atomic motion (mean velocity $v_{\mathrm{T}}$ ) at time $t=2 \Lambda /\left(\pi v_{\mathrm{T}}\right)$ after the initial excitation. Averaging over velocities results in decoherence of the collective state. (c) Atoms with different velocities experiencing different light shifts in the presence of a single dressing field, and spatial coherence maintained up to second order in velocity. The zeroth-order term introduces a global phase shift, which is evident when comparing the spin wave (purple) to its original form (gray). (d) Protection up to third order in velocity (thus covering a wider velocity range) when two dressing fields are applied, with no global phase shift introduced. Calculations are performed with $s=110$, $\Delta=4 v_{T} / \Lambda$, and optimal $\Omega$ from Eqs. (1) and (2). efficiency of light into the phase-matched direction $\eta(t)=$ $\eta(0) \mathcal{C}(t)[40]$. Here, $\eta(0)$ is the combined efficiency of the read and write processes. For pure motional dephasing, $\mathcal{C}(t)=e^{-\left(t / T_{\text {inhom }}\right)^{2}}$, where $T_{\text {inhom }}=\sqrt{2} / \sigma=\Lambda /\left(\sqrt{2} \pi v_{\mathrm{T}}\right)$ is the inhomogeneous dephasing time, and $v_{\mathrm{T}}$ is the atomic thermal velocity.

To apply the continuous protection scheme in the case of motional dephasing, we dress the spin wave with a sensor state that has large and opposite sensitivity to the velocity. This process is achieved by an optical transition whose wave vector is larger than $2 \pi / \Lambda$. The frequency shift of the transition $|\downarrow\rangle-|\uparrow\rangle$ due to the dressing field depends on the atom velocity via the Doppler effect and, for optimal dressing, exactly cancels the bare motional dephasing, rendering a velocity-insensitive state. Remarkably, as shown in Figs. 2(c) and 2(d), although the atoms are constantly in motion during the evolution of the state, the spin-wave correlations between position and phase are largely maintained. This unique time evolution is animated in Video 1 in the Supplemental Material [45].

Apart from protecting against dephasing of the spin wave (when $|W\rangle \rightarrow\left|W^{\prime}\right\rangle$ ), as quantified by the light retrieval efficiency, we are interested in protecting a general qubit $\alpha|G\rangle+\beta|W\rangle$. This qubit is formed, for instance, by mapping a photonic qubit $\alpha|0\rangle+\beta|1\rangle$, where $|0\rangle$ and $|1\rangle$ are photon number states, onto atomic collective states. Decoherence of $|W\rangle$ would naturally lead to decoherence of the qubit but so will global fluctuations of the relative phase of $\beta / \alpha$ [46]. The double-dressing scheme, which does not introduce a global phase that varies with drive noise, is therefore advantageous for robust protection of a qubit.

\section{EXPERIMENTAL REALIZATION}

To demonstrate and study the continuous protection against motional dephasing, we probe the coherence decay of a collective excitation in a hot atomic vapor, utilizing storage and retrieval of light. We employ the FLAME protocol [7] to store 1.8-ns (FWHM) signal pulses (with about 0.5 photons per pulse) as electronic-orbital excitations [Fig. 3(a)] in a 5-mm-long cell of thermal ${ }^{87} \mathrm{Rb}$ vapor at $98{ }^{\circ} \mathrm{C}$. The $|\uparrow\rangle$ and $|\downarrow\rangle$ states reside in the levels $5 S_{1 / 2}$ and $5 D_{5 / 2}$, respectively. The two states are coupled via a two-photon transition, detuned by $1.4 \mathrm{GHz}$ from the intermediate level $5 P_{3 / 2}[|i\rangle$ in Fig. 3(a)]. After some storage time $t$, we send a second control pulse and retrieve a signal pulse from the collective excitation [Fig. 3(c), top]. The memory efficiency $\eta(t) / \eta(0)$ is determined from the retrieved power (relative to the input power), and the temporal decay of the squared coherence is calculated from $\mathcal{C}(t)=\eta(t) / \eta(0)$. To measure the phase difference between incoming and retrieved signals, we store slightly longer signal pulses (4-ns FWHM) and interfere them with reference pulses shifted by $2 \pi \times 380 \mathrm{MHz}$, which do not traverse the atomic vapor [Fig. 3(c), bottom]. 
(a)

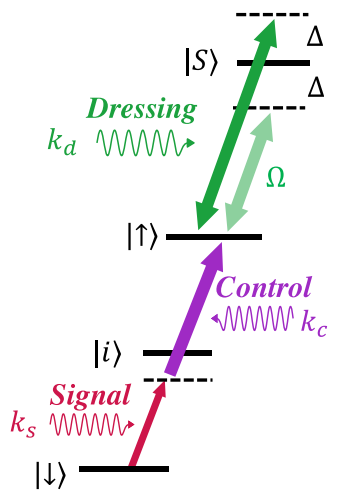

(b)

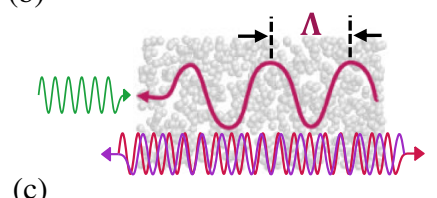

(c)

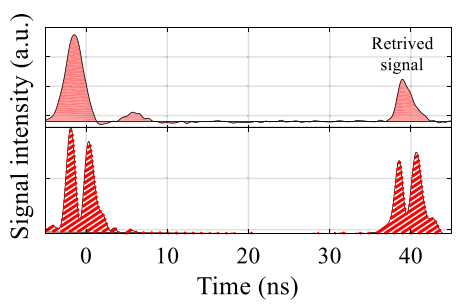

FIG. 3. Experimental realization of continuous protection from motional dephasing. (a) Light storage scheme: Counterpropagating pulses of a strong control field and a weak signal field with wave vectors $k_{\mathrm{c}}$ and $k_{\mathrm{s}}$ coupling the ground state $|\downarrow\rangle$ to the excited state $|\uparrow\rangle$ through an intermediate state $|i\rangle$. A dressing beam with wave vector $k_{\mathrm{d}}$ couples $|\uparrow\rangle$ to the sensor state $|S\rangle$ with Rabi frequency $\Omega$ and detuning $\Delta$. (b) The signal and control pulses generate a collective excitation with wave vector $2 \pi / \Lambda=k_{\mathrm{s}}-k_{\mathrm{c}}$. The dressing-field wave vector $k_{\mathrm{d}}$ has an opposite direction and a larger magnitude. (c) Typical data traces when the incoming and retrieved signals are either directly measured (top) or interfered with reference pulses to measure the phase of the signal (bottom).

The wave vectors for the signal and control transitions are, respectively, $k_{\mathrm{s}}=2 \pi / 0.78 \mu \mathrm{m}^{-1}$ and $k_{\mathrm{c}}=2 \pi /$ $0.776 \mu \mathrm{m}^{-1}$, giving an excitation wavelength $\Lambda=$ $2 \pi /\left(k_{\mathrm{s}}-k_{\mathrm{c}}\right)=151 \mu \mathrm{m}$ [Fig. 3(b)]. The Gaussian distribution of velocities with a thermal velocity $v_{T}=188 \mathrm{~m} / \mathrm{s}$ results in an inhomogeneous dephasing of the collective state over about 100 ns. Protection during storage is obtained by a field with wave vector $k_{\mathrm{d}}=2 \pi /$ $1.274 \mu \mathrm{m}^{-1}$, propagating along the direction of the signal field and weakly dressing the state $|\uparrow\rangle$ with the state $|S\rangle=28 F_{7 / 2}$. We use an electro-optic modulator (EOM) to generate the double-dressing configuration (see Appendix A). The sensor transition $|\downarrow\rangle-|S\rangle$ has an opposite and enhanced velocity sensitivity, $s=\left(k_{\mathrm{s}}-k_{\mathrm{c}}+k_{\mathrm{d}}\right) /$ $\left(k_{\mathrm{s}}-k_{\mathrm{c}}\right) \approx 110$, compared to that of the two-photon transition $|\downarrow\rangle-|\uparrow\rangle$, such that weak dressing is sufficient to form a velocity-insensitive collective state. The bare homogeneous decoherence rate of the collective state $\gamma=2 \pi \times 1.38 \mathrm{MHz}$ is limited by the $5 D_{5 / 2}$ radiative lifetime and by the transit time of atoms through the signal beam waist. In the presence of a dressing beam, we measure an increase of $\leq 25 \%$ in $\gamma$, which we attribute to resonant scattering due to imperfect extinction of the carrier frequency by the EOM and to residual excitation of atoms in the tail of the velocity distribution for small dressing-field detunings (see Appendix A).

To quantify the effect of protection, we compare the decay of coherence with no dressing field, with a single

dressing field, and with a double-dressing field; these cases are shown in Fig. 4. In the absence of dressing fields, the decay of efficiency with time has both exponential and Gaussian components, corresponding, respectively, to homogeneous and inhomogeneous dephasing. With a single dressing field, the inhomogeneous component is partially removed, while with the double-dressing field, it vanishes almost completely. As a result, we observe an increase of over an order of magnitude in retrieved signal at long storage times.

The residual inhomogeneous dephasing is revealed by normalizing the squared coherence $\mathcal{C}(t)$ by the homogeneous decoherence $e^{-\gamma t}$, as plotted in the inset of Fig. 4. With a double-dressing protection field, the inhomogeneous dephasing time is substantially elongated. Importantly, this implies that a dramatic improvement in the coherence times can be obtained for the broad range of systems that have a low homogeneous decoherence rate. The remaining, nonlinear dependence of the qubit transition frequency on the inhomogeneous shift $\delta$ results in non-Gaussian dephasing [47]. On long timescales, the coherence decays as a power law $\mathcal{C}(t) \propto t^{-2 / n}$, where $n$ is the first nonvanishing order of $\delta$ in the qubit transition frequency. However, on a short timescale, the dephasing is well approximated by a Gaussian decay.

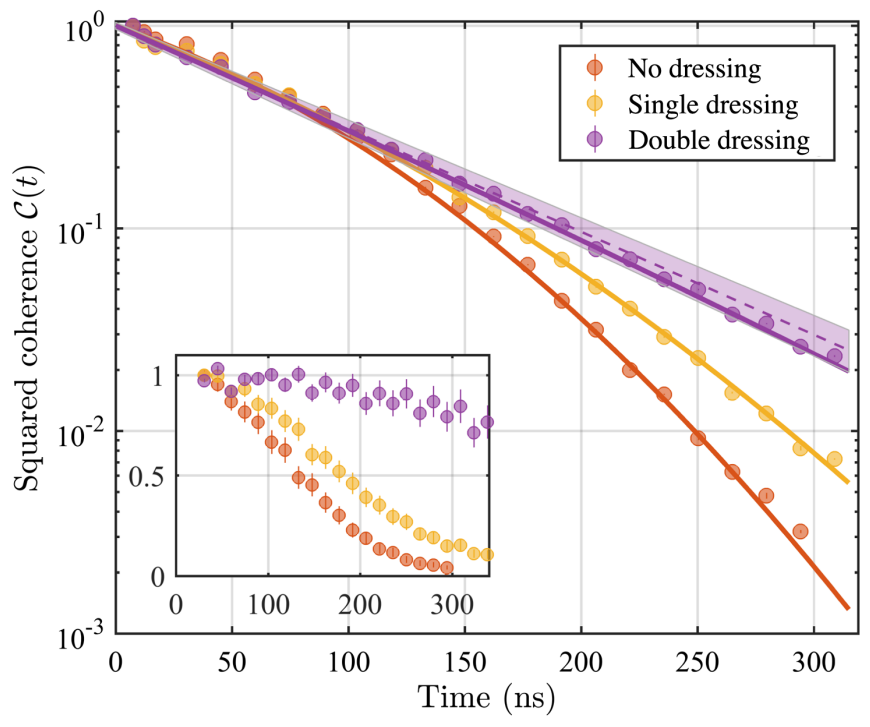

FIG. 4. Protection of a collective excitation from inhomogeneous dephasing. The squared coherence is calculated for different storage times by measuring the memory efficiency. Solid lines are fits to a model accounting for both homogeneous and inhomogeneous dephasing. With double dressing, the decay is predominantly homogeneous; the dashed line marks the homogeneous component of the fitted decay model, with the surrounding shaded area representing the fit uncertainty. Inset: inhomogeneous component of the decoherence $\mathcal{C}(t) / e^{-\gamma t}$, where the homogeneous component $e^{-\gamma t}$ is normalized out. The dressing-field parameters are $|\Delta|=2 \pi \times 350 \mathrm{MHz}, \Omega=2 \pi \times$ $34 \mathrm{MHz}$ (double dressing), and $\Omega=2 \pi \times 28 \mathrm{MHz}$ (single dressing). 
To further study the optimal conditions for continuous protection, we vary the dressing parameters $\Omega$ and $\Delta$ and measure the retrieval efficiency at $t=250 \mathrm{~ns}$, from which we infer the dephasing time $T_{\text {inhom }}$ (see Appendix A). Figure 5(a) shows the improvement in $T_{\text {inhom }}$ for the double-dressing protection scheme. The data agree with the condition $|\Delta|>s \sigma$ for efficient protection (here, $s \sigma=2 \pi \times 140 \mathrm{MHz}$ ). Substantial improvement in dephasing time is obtained over a large range of dressing powers and is further enhanced with larger detuning. For the largest detuning measured, we compare the data to the theoretical model (shaded gray line). Indeed, the improvement in $T_{\text {inhom }}$ is quadratic in $\Omega$ and reaches an optimal value at the protection condition (2), where it is limited by the third-order term in the inhomogeneous shift. The latter can be made negligible by increasing the detuning. We further validate the protection condition (2) in Fig. 5(b) by comparing the predicted ratio $\Omega / \Delta$ to the measured ratio $\Omega_{\text {opt }}(\Delta) / \Delta$. Excellent agreement is found for large detunings.

An important property of any protection or dynamical decoupling technique is the qubit phase introduced by the driving fields and its dependence on their intensities.
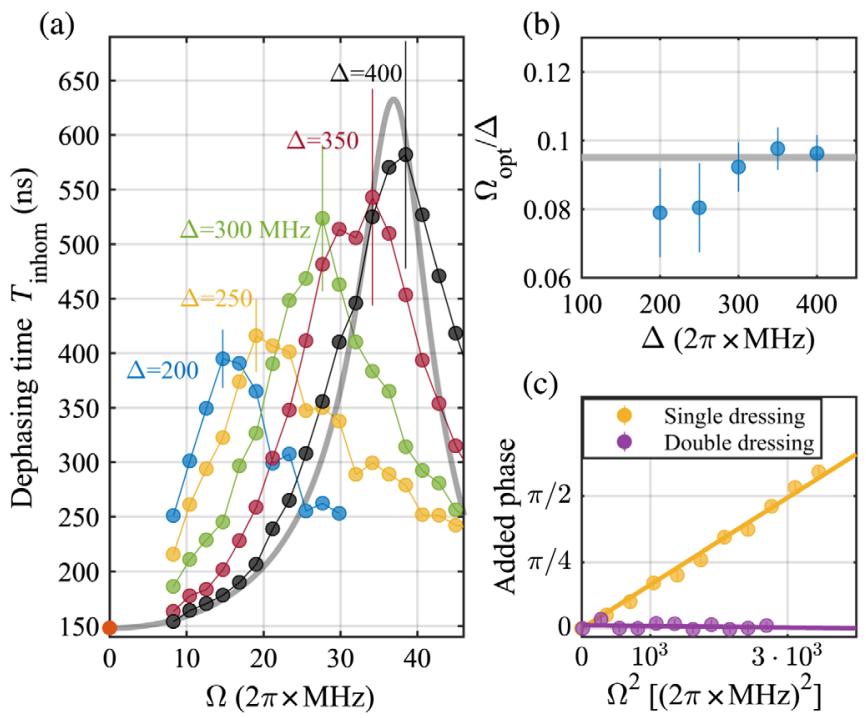

FIG. 5. Dependency of coherence time and accumulated phase on the dressing parameters. (a) Inhomogeneous dephasing time $T_{\text {inhom }}$, with double-dressing protection as a function of dressing Rabi frequency $\Omega$, for different detunings $\Delta$ (in $2 \pi \times \mathrm{MHz}$ ). The shaded gray line is a theoretical model, where the dephasing rates due to linear and cubic dependencies on $\delta$ are summed in quadrature. (b) Ratio $\Omega_{\text {opt }} / \Delta$, where $\Omega_{\text {opt }}$ is the Rabi frequency maximizing the gain for each $\Delta$, compared to the model prediction $2^{-3 / 2} J_{0}^{-1}[(s-1) /(s+1)] \approx s^{-1} \approx 0.095$ (solid line). (c) Phase accumulated due to the dressing fields during a storage time of $40 \mathrm{~ns}$. Here, $\Delta=2 \pi \times 480 \mathrm{MHz}$. Solid lines are linear fits. For double dressing, the added phase is zero (up to the experimental uncertainty) and is independent of the dressing intensity.
Measurements of this dependence for both single and double dressing are shown in Fig. 5(c). For single dressing, we find indeed that the phase shift scales linearly with the mean light shift $\Omega^{2} / \Delta$ [48], yielding a sensitivity to drive noise of about $0.4 \mathrm{mrad} /(2 \pi \times \mathrm{MHz})^{2}$. This result is in contrast to the double-dressing protection, where we measure essentially no added phase and no dependence on the dressing intensity or on the storage time.

\section{POTENTIAL APPLICATION TO OTHER SYSTEMS}

Motional dephasing occurs across various platforms, where it limits the performance of quantum information applications. One prominent example is that of neutral atoms trapped in tweezer arrays and interacting via Rydberg excitations. The coherence time and bell-state preparation fidelity in several recent realizations of this system are limited by motional dephasing $[9,49]$. Our scheme can be adapted to mitigate this effect by using a uniform field for globally dressing either the ground or the Rydberg state with an auxiliary metastable state or by implementing velocityinsensitive bichromatic entangling gates. An entirely different application is a single-photon source based on four-wave mixing in atomic vapor. Whether they rely on Rydberg interactions or not [50,51], the brightness and purity of these sources are limited as well by motional dephasing, which can be suppressed by implementing our scheme. Here, when it comes to stochastic events such as the heralded spontaneous generation of photons, a protection scheme that is continuous is essential. For collective Rydberg excitations, a threephoton excitation to the Rydberg state can be employed with an intermediate (two-photon) transition that is velocity sensitive (i.e., with larger sensitivity than in our experiment); this seemingly peculiar choice would yield a state that is protected from motional dephasing and, at the same time, has a large Rydberg admixing, such that it inherits the desired Rydberg-level qualities, namely, strong dipolar interactions.

Beyond the context of motional dephasing, our scheme can be applied to magnetically sensitive qubits, such as trapped ions and NV centers. Here, again, one should identify a sensor transition with an opposite and possibly larger magnetic moment difference relative to the qubit transition. For example, we suggest protecting an optical qubit in a ${ }^{88} \mathrm{Sr}^{+}$ion encoded in the $\left|S_{1 / 2} ; m_{j}=1 / 2\right\rangle-$ $\left|D_{5 / 2} ; m_{j}=1 / 2\right\rangle$ basis by dressing the ground state with the long-lived $\left|D_{5 / 2} ; m_{j}=-3 / 2\right\rangle$ state. This choice provides a sensitivity factor $s=6$ owing to the different Landé $g$-factors of the states involved and thus entails low admixing of the sensor level into the qubit state.

\section{DISCUSSION}

The presented protection scheme is akin to continuous dynamical decoupling but distinct in several aspects. Our protecting fields do not couple the two states comprising 
the qubit but rather couple one of them to an external state. They are preferably off resonant, and the overall protection improves with further detuning. In standard continuous dynamical decoupling, on the other hand, the protecting fields drive the qubit transition, often resonantly, exposing it directly to drive noise $\delta_{\Omega}$ when the field amplitude fluctuates, $\Omega \rightarrow \Omega+\delta_{\Omega}$. Moreover, if $\delta$ fluctuates in time, the fluctuation bandwidth over which the protection is effective is limited by the available Rabi frequency [52]. In our scheme, the bandwidth is fundamentally limited by the detuning (or the effective Rabi frequency $\sqrt{4 \Omega^{2}+\Delta^{2}}$ ), which can be made much larger given the same limited power, especially with large sensitivity $s$ (for which $\Delta \gg \Omega$ ).

In Table I, we present the effectiveness of our protection schemes in terms of the residual (higher-order) sensitivities to inhomogeneous shifts $\mathrm{O}\left(\delta^{2}\right)$ and $\mathrm{O}\left(\delta^{3}\right)$, to Rabi frequency fluctuations $\mathrm{O}\left(\delta_{\Omega}\right)$, and to cross terms of the two $\mathrm{O}\left(\delta \delta_{\Omega}\right)$. For a single dressing field, an enhanced sensitivity $s \gg 1$ earned from utilizing a sensor state enables the use of a protection field that is far detuned, thus reducing the sensitivity to drive noise to $\delta_{\Omega} / \sqrt{s}$. Further suppression of drive noise is achieved with double dressing, which we find to be insensitive to drive noise to leading order $\mathrm{O}\left(\delta_{\Omega}\right)$ [also supported by the measurements in Fig. 5(c)]. Our analysis additionally shows that the double dressing removes sensitivity to inhomogeneous shifts up to $\mathrm{O}\left(\delta^{3}\right)$. The remaining sensitivity scales inversely with Rabi frequency or with detuning, but we note that it scales unfavorably with the sensitivity factor $s$. The dephasing time $T_{\text {inhom }}$ is proportional to the inverse of the leading residual sensitivity term when substituting $\delta=\sigma$.

For a sensor state with sensitivity equal to that of the qubit state, $s=1$, the single-dressing protection condition [Eq. (1)] requires a resonant field $\Delta=0$, which results in two, equally mixed, protected states $[42,43]$. The doubledressing scheme also generates two protected states in this case (see Appendix B and Fig. 7), without the need for resonant driving. The $s=1$ case resembles the case of a two-level system, where the condition (2) provides for intrinsic dynamical decoupling in bichromatic entangling gates with trapped ions [53].

TABLE I. Leading residual sensitivities of the qubit transition frequency: first order in the drive noise $\left(\delta_{\Omega}\right)$, second and third order in the inhomogeneous shift $\left(\delta^{2}, \delta^{3}\right)$, and the cross term $\left(\delta_{\Omega} \cdot \delta\right)$. For the double-dressing scheme, exact numerical factors depend on the experimental realization, including the phase between the two fields. These factors and their exact derivation are given in Appendix B.

\begin{tabular}{|c|c|c|c|c|c|c|}
\hline \multicolumn{3}{|c|}{ Single dressing } & \multicolumn{4}{|c|}{ Double dressing } \\
\hline & $\mathrm{O}\left(\delta_{\Omega}\right)$ & $\mathrm{O}\left(\delta^{2}\right)$ & $\mathrm{O}\left(\delta_{\Omega}\right)$ & $\mathrm{O}\left(\delta^{2}\right)$ & $\mathrm{O}\left(\delta \delta_{\Omega}\right)$ & $\mathrm{O}\left(\delta^{3}\right)$ \\
\hline$s=1$ & $\delta_{\Omega}$ & $\frac{1}{2}\left(\delta^{2} / \Omega\right)$ & 0 & 0 & $\sim 1\left(\delta \delta_{\Omega} / \Omega\right.$ & $\frac{1}{2}\left(\delta^{3} / \Omega^{2}\right)$ \\
\hline$s \gg 1$ & $(2 / \sqrt{s}) \delta_{\Omega}$ & $\sqrt{s}\left(\delta^{2} / \Omega\right)$ & 0 & 0 & $2\left(\delta \delta_{\Omega} / \Omega\right.$ & $S\left(\delta^{3} / \Omega^{2}\right)$ \\
\hline
\end{tabular}

In conclusion, we have introduced and demonstrated a new scheme for protection from inhomogeneous dephasing, which is continuous, efficient, and robust to drive noise. The scheme is particularly suitable for protection from motional dephasing of a collective state stored in an atomic ensemble, where limited solutions have been suggested to date. The minimal requirements outlined in this paper may be found across numerous systems where a multilevel structure exists. We have discussed a few examples for such systems, where this scheme can be applied to enhance the performance of quantum sensors, sources, and gates.

Our experimental demonstration confirms the validity of the scheme by eliminating the inhomogeneous dephasing of a collective excitation in a gas of thermal atoms. Remarkably, this elimination is achieved not through time reversal of the process of dephasing or of the direction of atomic motion, but through continuously maintaining the original position-dependent phases of atoms that randomly move to different positions.

The compatibility of this scheme to various protocols of quantum information processing, including gates and metrology, requires further research. It could provide an important tool for applications operating with either singleconstituent qubits or with collective quantum states of ensembles.

\section{ACKNOWLEDGMENTS}

We acknowledge financial support by the Israel Science Foundation and ICORE, the European Research Council starting investigator grant QPHOTONICS 678674, the Pazy Foundation, the Minerva Foundation with funding from the Federal German Ministry for Education and Research, a BSF-NSF research grant, and the Laboratory in Memory of Leon and Blacky Broder. I. C. acknowledges support from Marie Skodowska-Curie Grant Agreement No. 785902.

R.F., O.L., O.D., S. K., and E.P. contributed to the experimental design, construction, data collection, and analysis of this experiment. I. C., R.F., O. L., and E.P. developed the theoretical framework supporting the experiment. E. P. and O.F. supervised the entire project. All authors discussed the results and contributed to writing the manuscript.

\section{APPENDIX A: EXPERIMENTAL METHODS}

\section{Experimental design}

The setup comprises a 780-nm-distributed Bragg reflector (DBR) diode laser, serving as the signal beam and a 776-nm external cavity diode laser (ECDL) amplified by a tapered amplifier (TA), serving as the control beam. The signal laser is offset locked to a master ultrastable fiber laser using a fast beat-note detector. The control laser is locked to a two-photon absorption feature in a reference cell when 
overlapped with the master laser, where the latter is frequency shifted by a fiber electro-optic phase modulator (EOPM). The signal field is amplitude modulated in time by two fiber electro-optic amplitude modulators (EOAM) to carve a Gaussian pulse of 1.8-ns FWHM, with a combined extinction ratio of $1: 3000$. The control field is amplitude modulated by two Pockels cells (PCs), generating pulses of 2.5-ns FWHM with an extinction ratio of $1: 1000$. The repetition rate of the experiment is set by that of the PCs to $100 \mathrm{kHz}$. After the modulators, the control beam is passed through a tilted filter (Semrock LL01-78012.5) functioning as a 776-nm bandpass, filtering out other frequencies that might be produced in the TA. Both beams are passed through single-mode fibers (SMF), aligned with each other in a counterpropagating geometry, and overlapped at the center of the vapor cell. The signal beam is focused down to $w_{0}=85 \mu \mathrm{m}$, while the control beam is focused down to $w_{0}=200 \mu \mathrm{m}$. Both beams are $\sigma^{+}$ polarized, and we optically pump all atoms to the stretched state, a combination that guarantees purely orbital transitions and greatly simplifies the multilevel structure of the atomic vapor. The optical pumping is realized by a "pump" and a "repump" at $795 \mathrm{~nm}$, both $\sigma^{+}$polarized, and separated from each other by $6.8 \mathrm{GHz}$, such that the pump (repump) is resonant with the $F=2 \rightarrow F^{\prime}=2$ $\left(F=1 \rightarrow F^{\prime}=2\right)$ transition of the rubidium D1 line. The pump (repump) beam power at the vapor cell is $300 \mathrm{~mW}(200 \mathrm{~mW})$. The pump beams are $1.2 \mathrm{~mm}$ wide and are directed at a small angle with respect to the control beam. The 5 -mm-long ${ }^{87} \mathrm{Rb}$ vapor cell is antireflection coated for $780-1064 \mathrm{~nm}$. It is heated to $72{ }^{\circ} \mathrm{C}$ at its coldest spot and $98^{\circ} \mathrm{C}$ at its hottest spot using two electrical current heaters, to set a $\mathrm{Rb}$ density of $6.5 \times 10^{11} \mathrm{~cm}^{-3}$ and an optical depth $\mathrm{OD} \approx 5$. We obtain $\mathrm{OD} \approx 8.5$ with continuous optical pumping. After the cell, the signal beam is passed through a polarizing beam splitter and two 780-nm bandpass filters to filter out any residual 776-nm and 795-nm components. It is then coupled to a SMF acting as a spatial filter, removing most of the spatially incoherent fluorescence emitted from the cell at $780 \mathrm{~nm}$. The SMF is coupled either to a fast linear avalanche photodetector (APD) with a bandwidth of $1 \mathrm{GHz}$ or to a single-photon counting module (SPCM) connected to a time tagger with time bins of $100 \mathrm{ps}$.

\section{Dressing-field generation}

The dressing beam is produced by a 1274-nm ECDL and is then passed through both an EOAM and an EOPM. For the double-dressing protection scheme, the EOPM is modulated by a square-wave electronic signal (70-ps rise or fall time), generating dominant first-order side bands comprising $78 \%$ of the total output power and a vanishingly small carrier $(0.2 \%)$. The dressing beam is then amplified by an O-band booster optical amplifier (Thorlabs BOA1130S) and further amplified by a TA. The dressing laser frequency is stabilized to a wavelength meter with 1-MHz resolution (High-Finesse WS8-IR1). The dressing beam is combined with the signal beam on a dichroic mirror and focused to $w_{0}=200 \mu \mathrm{m}$ in the vapor cell. It is also $\sigma^{+}$polarized, thus coupling only to the stretched state.

\section{Homogeneous decoherence in the experiment}

With a single dressing field and, more significantly, with a double-dressing field, we measure a small increase in the homogeneous decoherence rate (of up to 25\%). The first contribution to this increase is the scattering rate of atoms at the tail of the inhomogeneous distribution. For the intermediate detunings we use in the experiment, $|\Delta| \lesssim 3 \sigma$, there is a small but nonvanishing fraction of atoms whose transition frequency is near resonant with the dressing field. The overall scattering rate due to this contribution is on the order of 1.2-6.3 $\times 10^{6} \mathrm{~s}^{-1}$ in the range of parameters used in the experiment and scales inversely with $\Delta^{2}$.

For the double-dressing scheme, the experimental realization (as detailed above) includes a single laser tuned to resonance and a fast square-wave phase modulation, whose imperfections result in a carrier intensity of up to $\Omega_{\text {carrier }}^{2} / \Omega^{2}=0.3(2) \%$. This case corresponds to a calculated resonant scattering rate of up to $0.6 \times 10^{5} \mathrm{~s}^{-1}$ (at the optimal Rabi frequency of $\Omega=2 \pi \times 35 \mathrm{MHz}$ ).

\section{Evaluation of $\boldsymbol{T}_{\text {inhom }}$}

To obtain the inhomogeneous dephasing time, we measure the memory efficiency for a long storage time of $250 \mathrm{~ns}$. The results of this measurement are presented in Fig. 6(a). We extract $T_{\text {inhom }}$ from the efficiency by fitting to a Gaussian decay model $e^{-\left(t / T_{\text {inhom }}\right)^{2}}$ after normalizing out the homogeneous decoherence $e^{-\gamma t}$. We include the slight increase in $\gamma$ in the presence of the dressing field by measuring $\gamma$ for several dressing powers at $\Delta=$ $2 \pi \times 350 \mathrm{MHz}$ (as in Fig. 4 in the main text) and calibrate our model for resonant scattering $\gamma=\gamma_{0}+\Gamma \Omega^{2} / \Delta^{2}$ accordingly. We verify the validity of the model across a wide range of dressing-field parameters as presented in Fig. 6. Larger available laser power and faster modulation of the dressing field would enable operation at larger detunings, which would greatly suppress such scattering.

\section{Phase measurements}

In order to measure the phase between the incoming and retrieved signals, we generate two consecutive signal pulses and pass them through an acousto-optical modulator (AOM). The diffracted pulses, shifted by $2 \pi \times 380 \mathrm{MHz}$ relative to the signal, will act as a reference. They are thus coupled into an optical fiber acting as a delay line. The time difference between the consecutive pulses, the memory storage time, and the time delay in the delay line are all set to be identical. This method allows part of the incoming signal, which was not stored due to limited efficiency, to 
(a)

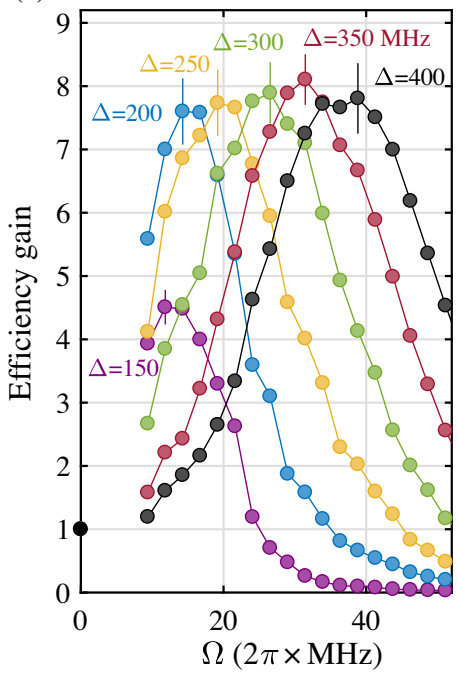

(b)

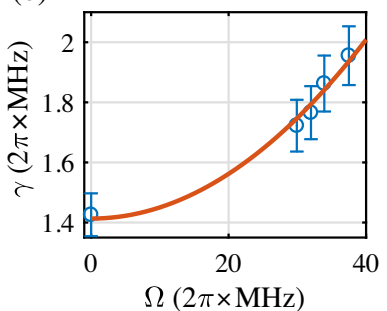

(c)

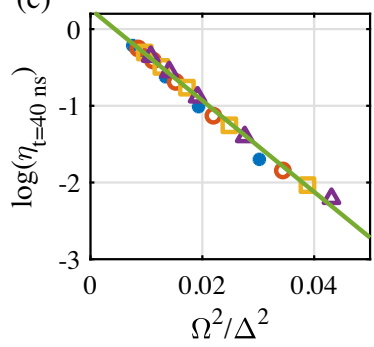

FIG. 6. Efficiency enhancement and loss with a dressing field at intermediate detunings. (a) Gain in efficiency at a long storage time $(t=250 \mathrm{~ns})$ with double-dressing protection as a function of dressing Rabi frequency $\Omega$. Solid lines are a guide to the eye. The gain saturates due to a slight increase in homogeneous decoherence at large dressing powers. (b) Added homogeneous decoherence rate $\gamma$ for several dressing Rabi frequencies $\Omega$ at detuning $\Delta=2 \pi \times 350 \mathrm{MHz}$. The solid line is a fit to a quadratic model. (c) Efficiency loss at a short storage time $(t=40 \mathrm{~ns})$ for different powers (marked as different markers) and for several detunings. All data sets collapse when plotted against $\Omega^{2} / \Delta^{2}$ and fit well to the scattering model $\gamma=\gamma_{0}+\Gamma \Omega^{2} / \Delta^{2}$ across a wide range of the parameter $\Omega / \Delta$.

interfere with the first reference pulse and the retrieved signal to interfere with the second reference pulse. The phase difference between these two pulses, acquired in a single shot within tens of ns, is thus insensitive to interferometer drifts, which occur over a much longer timescale. We then average this value over hundreds of repetitions.

\section{APPENDIX B: DERIVATION OF PROTECTION CONDITIONS AND SENSITIVITY ANALYSIS}

Let $\{|\downarrow\rangle,|\uparrow\rangle,|S\rangle\}$ be a three-level system, comprising a qubit $\{|\downarrow\rangle,|\uparrow\rangle\}$ and a sensor state $|S\rangle$. Let the frequencies of the transitions $|\downarrow\rangle-|\uparrow\rangle$ and $|\downarrow\rangle-|S\rangle$ be inhomogeneously shifted, with the magnitude of the shifts linearly dependent on the same inhomogeneous variable $\delta$. We assume (without loss of generality—see [54]) that $|\downarrow\rangle-|\uparrow\rangle$ and $|\downarrow\rangle-|S\rangle$ shift to opposite directions and that $|\downarrow\rangle-|S\rangle$ is at least as sensitive as $|\downarrow\rangle-|\uparrow\rangle$ to the inhomogeneity. Hereafter, we take the ground state $|\downarrow\rangle$ as the frequency reference and examine the transition frequencies relative to it. In a frame rotating with the unshifted frequencies, the Hamiltonian in the subspace $\{|\uparrow\rangle,|S\rangle\}$ is given for any constituent in the ensemble as $(\hbar=1)$

$$
H_{0}=\delta\left(\begin{array}{cc}
-1 & 0 \\
0 & s
\end{array}\right)
$$

Here, $\delta$ is a random (inhomogeneous) variable with a standard deviation $\sigma$, and the sensitivity parameter $s \geq 1$ is defined by construction. We aim to add coupling fields in this subspace that will render (at least one) protected state, i.e., a dressed state with a reduced sensitivity to $\delta$. Note that we do not explicitly use $\sigma$ in the following; rather, it is implicitly understood that $\sigma$ should be substituted for $\delta$ when assessing the validity of various expansions in leading orders in $\delta$.

\section{Protection by a single-tone dressing field}

A single classical field that dresses the transition $|\uparrow\rangle-$ $|S\rangle$ with a Rabi frequency $\Omega$ and detuning $\Delta$ yields the Hamiltonian

$$
H=H_{0}+\left(\begin{array}{cc}
\Delta & \Omega \\
\Omega & 0
\end{array}\right) .
$$

By diagonalizing $H$, we obtain the new transition frequencies

$\omega_{ \pm}=\frac{1}{2}\left[\Delta+\delta(s-1) \pm \sqrt{[\Delta+(s+1) \delta]^{2}+4 \Omega^{2}}\right]$.

By requiring a vanishing derivative with respect to $\delta$ of one of the frequencies $\left.(d \omega / d \delta)\right|_{\delta \rightarrow 0}=0$, we obtain the protection condition [Eq. (1) in the main text]

$$
\left(\frac{\Omega}{\Delta}\right)^{2}=\frac{s}{(s-1)^{2}}
$$

In the case of a sensor state with high sensitivity $s \gg 1$, this condition reduces to $(\Omega / \Delta)^{2}=1 / s$.

An example for the spectrum of the two dressed states under the protection condition is shown in Fig. 7. For the general case $s \neq 1$, the two states $|\uparrow\rangle,|S\rangle$ are not equally mixed, and we obtain a single protected state. Conversely, when $s=1$, optimal protection is reached with a resonant dressing $\Delta=0$ and with the two states equally mixed; therefore, we obtain two protected states, which can be employed to form a qutrit. In this regime, our scheme is akin to the traditional two-level continuous dynamical decoupling (CDD) [55].

While protecting up to first order in $\delta$, the single dressing field adds a qubit transition frequency shift of second order in the inhomogeneous shift $\delta$,

$$
\Delta \omega_{\delta^{2}}=\frac{s \delta^{2}}{\Omega} \frac{\sqrt{s}}{s+1},
$$

which is inversely proportional to the available $\Omega$. In addition, the protection is subjected to drive noise in the form of amplitude fluctuations $\Omega \rightarrow \Omega+\delta_{\Omega}$, giving rise to a transition frequency shift 

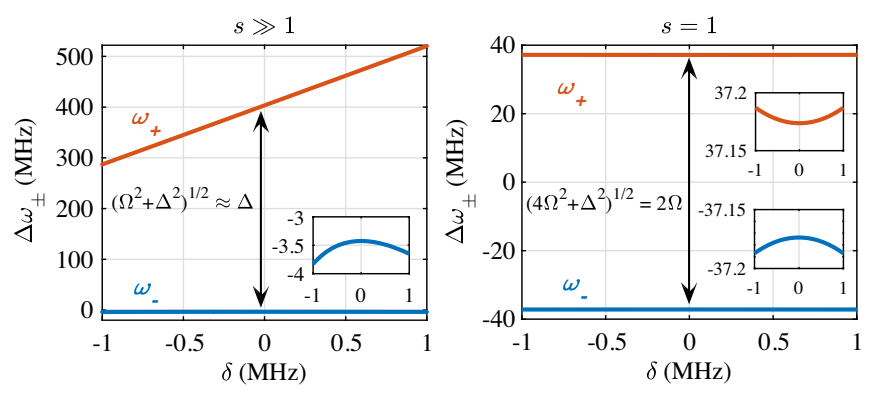

FIG. 7. Dressed-state spectrum for a single-dressing protection. Transition frequency shift as a function of the inhomogeneous shift $\delta$, after introducing a dressing field with Rabi frequency $\Omega=37 \mathrm{MHz}$ and detuning $\Delta$. Left panel: For high sensor sensitivity $(s=110 \gg 1)$, there exists one protected state (insensitive to $\delta$ to first order). The energy gap is dominated by the detuning $\Delta=400 \mathrm{MHz}$, which is much larger than $\Omega$. Right panel: For $s=1$, there exist two protected states. Optimal protection is achieved with a resonant dressing field $(\Delta=0)$, and thus, the energy gap is dominated by $\Omega$. Insets magnify the spectrum near $\delta=0$, revealing the magnitude of the secondorder shift.

$$
\Delta \omega_{\delta_{\Omega}}=2 \delta_{\Omega} \frac{\sqrt{s}}{s+1} .
$$

For high sensitivity $s \gg 1$, the drive noise is attenuated by $1 / \sqrt{s}$, while the second-order inhomogeneous shift is enhanced by $\sqrt{s}$. These scalings are summarized in Table II (left section) and compared to the residual transition frequency shifts under the other protection schemes, presented in the following sections.

\section{Protection by a two-tone dressing field}

A dressing field comprising two tones with symmetric detuning $\pm \Delta$ around the $|\uparrow\rangle-|S\rangle$ transition can protect the $|\downarrow\rangle-|\uparrow\rangle$ transition better than a single-tone dressing, that is, up to higher orders in the inhomogeneous shift $\delta$ and in the drive noise $\delta_{\Omega}$. With this field, the timedependent Hamiltonian in the rotating frame is given by $H(t)=H_{0}+H_{2}(t)$, where

$$
H_{2}(t)=\sqrt{2} \Omega \cos (\Delta t+\phi)\left(\begin{array}{ll}
0 & 1 \\
1 & 0
\end{array}\right),
$$

where $\phi$ is the phase between the two tones of the dressing at $t=0$. The $\sqrt{2}$ factor appears above in order to maintain the overall dressing power as in the single-dressing scheme.

\section{a. Solution for $s \gg 1$}

Before presenting the general solution, we consider the limit $s \gg 1$. Since $\Delta \gg \Omega$, we can solve the time-dependent Hamiltonian using the effective Hamiltonian technique [56], where each field gives rise to a standard light shift,

$$
\begin{aligned}
H_{ \pm \Delta} & =\frac{\Omega^{2} / 2}{ \pm \Delta+\delta(s+1)}\left(\begin{array}{cc}
-1 & 0 \\
0 & 1
\end{array}\right) \\
& \approx \frac{\Omega^{2}}{ \pm 2 \Delta}\left(1-\frac{s \delta}{ \pm \Delta}+\left[\frac{s \delta}{\Delta}\right]^{2}\right)\left(\begin{array}{cc}
-1 & 0 \\
0 & 1
\end{array}\right) .
\end{aligned}
$$

When summing both contributions $H \approx H_{+\Delta}+H_{-\Delta}$, we are left with only the odd orders in $\delta$. The first order compensates for the inhomogeneous shift terms in the bare Hamiltonian $H_{0}$ when the protection condition

$$
\frac{\Omega^{2}}{\Delta^{2}}=\frac{1}{s}
$$

is satisfied. This condition coincides with that of the singledressing scheme [Eq. (B4)] for $s \gg 1$. However, unlike for single dressing, here the residual inhomogeneity is of third order in $\delta$, as both the first and the second orders are eliminated. In addition, since the zeroth order of the light shift is eliminated as well, the double-dressing scheme is also protected from the first-order contribution of the drive noise $\delta_{\Omega}$ (the fluctuations in $\Omega$ ). A general solution and a complete sensitivity analysis for the general case are presented below.

\section{b. Solution for general $s$ using the Magnus expansion}

We use the Magnus expansion to calculate both the exact protection condition for a general $s$ and the remaining highorder noise contributions. We start by writing the bare Hamiltonian (B1) as

$$
H_{0}=\frac{\delta}{2}\left([s-1] \mathbb{I}-[s+1] \sigma_{z}\right),
$$

where $\sigma_{z}$ is the Pauli- $z$ operator and $\mathbb{I}$ is the identity. To ease the derivation, we rotate the system around the $y$ axis, such

\begin{tabular}{|c|c|c|c|c|c|c|c|c|c|c|}
\hline & \multicolumn{2}{|c|}{ Single-tone dressing } & \multicolumn{4}{|c|}{ Two-tone dressing } & \multicolumn{4}{|c|}{ Stepwise phase } \\
\hline & $\mathrm{O}\left(\delta_{\Omega}\right)$ & $\mathrm{O}\left(\delta^{2}\right)$ & $\mathrm{O}\left(\delta_{\Omega}\right)$ & $\mathrm{O}\left(\delta^{2}\right)$ & $\mathrm{O}\left(\delta \delta_{\Omega}\right)$ & $\mathrm{O}\left(\delta^{3}\right)$ & $\mathrm{O}\left(\delta_{\Omega}\right)$ & $\mathrm{O}\left(\delta^{2}\right)$ & $\mathrm{O}\left(\delta \delta_{\Omega}\right)$ & $\mathrm{O}\left(\delta^{3}\right)$ \\
\hline $\begin{array}{l}s=1 \\
s \gg 1\end{array}$ & $\begin{array}{c}\delta_{\Omega} \\
(2 / \sqrt{s}) \delta_{\Omega}\end{array}$ & $\begin{array}{l}\frac{1}{2}\left(\delta^{2} / \Omega\right) \\
\sqrt{s}\left(\delta^{2} / \Omega\right)\end{array}$ & $\begin{array}{l}0 \\
0\end{array}$ & $\begin{array}{l}0 \\
0\end{array}$ & $\begin{array}{c}1.25\left(\delta \delta_{\Omega} / \Omega\right) \\
2\left(\delta \delta_{\Omega} / \Omega\right)\end{array}$ & $\begin{array}{c}(0.06 \sim 0.4)\left(\delta^{3} / \Omega^{2}\right) \\
s\left(\delta^{3} / \Omega^{2}\right)\end{array}$ & $\begin{array}{l}0 \\
0\end{array}$ & $\begin{array}{l}0 \\
0\end{array}$ & $\begin{array}{c}\delta \delta_{\Omega} / \Omega \\
2\left(\delta \delta_{\Omega} / \Omega\right)\end{array}$ & $\begin{array}{c}\frac{1}{2}\left(\delta^{3} / \Omega^{2}\right) \\
(6 s / 5)\left(\delta^{3} / \Omega^{2}\right)\end{array}$ \\
\hline
\end{tabular}
that $\sigma_{x} \rightarrow S_{z}$ and $\sigma_{z} \rightarrow-S_{x}$. The rotated Hamiltonian is

$$
\begin{aligned}
H_{y} & =U_{y}\left(H_{0}+H_{2}\right) U_{y}^{\dagger} \\
& =\frac{\delta}{2}\left[(s-1) \mathbb{I}+(s+1) S_{x}\right]+\sqrt{2} \Omega S_{z} \cos (\Delta t+\phi) .
\end{aligned}
$$

TABLE II. Higher-order corrections for the qubit transition frequency shifts: first-order in the drive noise $\left[\mathrm{O}\left(\delta_{\Omega}\right)\right]$, second- and thirdorder in the inhomogeneous shift $\left[\mathrm{O}\left(\delta^{2}\right), \mathrm{O}\left(\delta^{3}\right)\right]$, and their cross term $\left[\mathrm{O}\left(\delta \delta_{\Omega}\right)\right]$. 
Next, we move to a time-dependent rotating frame, utilizing $\mathrm{H}_{2}(t)$ to construct the unitary rotation operator

$$
\begin{aligned}
U(t) & =\exp \left(-\frac{i}{\hbar} \int_{0}^{t} U_{y} H_{2}(t) U_{y}^{\dagger} d t\right) \\
& =\exp \left[-i S_{z} \sqrt{2}(\Omega / \Delta)(\sin (\Delta t+\phi)-\sin \phi)\right]
\end{aligned}
$$

and obtain the Hamiltonian in the interaction picture

$H_{y}^{\prime}=\frac{\delta}{2}\left[(s-1) \mathbb{I}+(s+1)\left(S_{+} e^{i z[\sin (\Delta t+\phi)-\sin \phi]}+\right.\right.$ H.c. $\left.)\right]$,

with $z=2 \sqrt{2} \Omega / \Delta$.

Using the Jacobi-Anger expansion

$$
e^{ \pm i z \sin (\Delta t+\phi)}=\sum_{n=-\infty}^{\infty} J_{n}(z) e^{ \pm i n(\Delta t+\phi)},
$$

we expand Eq. (B13) in leading orders of the Magnus expansion. The first order reads

$$
\begin{aligned}
H^{(1)} & =\frac{1}{t} \int_{0}^{t} H_{y}^{\prime}\left(t_{1}\right) d t_{1} \\
& =\frac{\delta}{2}\left[(s-1) \mathbb{I}+J_{0}(z)(s+1)\left(S_{+} e^{-i z \sin \phi}+S_{-} e^{i z \sin \phi}\right)\right] .
\end{aligned}
$$

Since $\left(S_{+} e^{-i z \sin \phi}+S_{-} e^{i z \sin \phi}\right)$ is a sum of Pauli matrices, it has two eigenvalues \pm 1 . To find the protection condition [Eq. (2) in the main text], we require that the eigenvalues of $H^{(1)}$ be independent of $\delta$, which occurs when

$$
J_{0}(z)=\frac{s-1}{s+1} .
$$

For $s \gg 1$, the condition (B16) can be expanded to the leading order in $z$ :

$$
1-\frac{z^{2}}{4}+O\left(z^{4}\right)=1-\frac{2}{s}+O\left(s^{-2}\right)
$$

where we recover the previous (light shift) condition (B9). For $s=1$, as before, the first-order dependence on $\delta$ vanishes for both dressed states, and we obtain two protected states. Therefore, again, the whole three-level system is protected $[53,57]$.

For a general $s \neq 1$, the protected dressed state is rotated by $z \sin \phi$ around the $x$ axis in the $\{|\uparrow\rangle,|S\rangle\}$ subspace,

$$
|P\rangle=\exp \left(-i \sigma_{x} \frac{z \sin \phi}{2}\right)|\uparrow\rangle .
$$

When the fields are in phase $(\phi=0)$, or for high sensitivity $s \gg 1$ (as in our experiment), the dressed state is approximately the bare state $|\uparrow\rangle$. Otherwise, in order to efficiently couple to the protected dressed state, the phase term $(z \sin \phi) / 2$ needs to be well defined, and it would determine the adiabatic condition for switching on and off of the protection fields [58].

\section{c. Higher-order transition frequency shift contributions}

To find the higher-order noise terms, it is beneficial to make the distinction between noise terms that are parallel and perpendicular to the dressed-state basis. For brevity, we define the Pauli- $x$ operator in the $z \sin \phi$-dependent (dressed state) basis as $F_{x}=\left(S_{+} e^{-i z \sin \phi}+S_{-} e^{i z \sin \phi}\right)$ and rewrite Eq. (B15) as

$$
H^{(1)}=\frac{\delta(s-1)}{2}\left(\mathbb{I}+F_{x}\right) .
$$

The protected dressed state is the eigenstate of $F_{x}$ with the eigenvalue -1 . The perpendicular Pauli operators are $F_{y}=$ $-i\left(S_{+} e^{-i z \sin \phi}-S_{-} e^{i z \sin \phi}\right)$ and $F_{z}=S_{z}$.

We begin with the cross term of the inhomogeneous shift $\delta$ and the drive amplitude fluctuations $\delta_{\Omega}$,

$H_{\delta \cdot \delta_{\Omega}}^{(1)}=\left[-J_{1}(z) F_{x}+\sin \phi J_{0}(z) F_{y}\right] \frac{2 \sqrt{2}}{\Delta} \frac{s+1}{2} \delta \delta_{\Omega}$.

With respect to Eq. (B19), this noise term has both a parallel $\left(\propto F_{x}\right)$ and a perpendicular $\left(\propto F_{y}\right)$ contribution. Notably, the perpendicular contribution vanishes when $\phi \rightarrow 0$. For a general $\phi$, we examine the limits $s=1$ and $s \gg 1$ :

(i) For $s=1$, according to condition (B16), we set $J_{0}(z)=(s-1) /(s+1)=0$ and are left with only the parallel term

$$
\Delta \omega_{\delta \cdot \delta_{\Omega}} \approx 1.25 \frac{\delta \delta_{\Omega}}{\Omega} .
$$

(ii) For $s \gg 1$, we approximate the perpendicular term as $H_{\delta \cdot \delta_{\Omega}, \perp}^{(1)} \approx \sin \phi \sqrt{2 s} \delta\left(\delta_{\Omega} / \Omega\right) F_{y}$. From Eq. (B19), if the energy gap $\Delta \omega=\delta(s-1)$ in the $F_{x}$ direction is larger than the perpendicular $F_{y}$ noise, and assuming $\sin \phi \sqrt{2 / s}\left(\delta_{\Omega} / \Omega\right) \ll 1$, then the perpendicular noise manifests only as a small perturbation $\Delta \omega_{\delta \cdot \delta_{\Omega}, \perp} \propto \delta\left(\delta_{\Omega} / \Omega\right)^{2}$. Therefore, the leading contribution is the parallel term

$$
\Delta \omega_{\delta \cdot \delta_{\Omega}}=2 \frac{\delta \delta_{\Omega}}{\Omega}
$$

We now evaluate the residual second-order contribution of the inhomogeneous shift. We take the next order in the Magnus expansion using the Jacobi-Anger expansion (B14)

$$
\begin{aligned}
H^{(2)} & =-\frac{i}{2 t} \int_{0}^{t} d t_{1} \int_{0}^{t_{1}} d t_{2}\left[H_{y}^{\prime}\left(t_{1}\right), H_{y}^{\prime}\left(t_{2}\right)\right] \\
& =-2\left[\frac{\delta(s+1)}{2}\right]^{2} J_{0}(z) \sum_{n \neq 0} \frac{J_{n}(z) \cos n \phi}{n \Delta} F_{z}
\end{aligned}
$$

and again examine different $s$ regimes:

(i) For $s=1$, we have $J_{0}(z)=0$, and therefore $H^{(2)}=0$ for any $\phi$. 
(ii) For general $s>1, H^{(2)}$ contains a perpendicular $\left(F_{z}\right)$ contribution with respect to Eq. (B19), which vanishes for $\phi \rightarrow \pi / 2$. For a general $\phi$, this perpendicular term is not vanishing.

(iii) In the limit $s \gg 1$ and $z / 2=\sqrt{2 / s} \ll 1$, we expand $H^{(2)}$ to leading orders,

$$
\begin{aligned}
H^{(2)} & \approx-\left[\frac{\delta(s+1)}{2}\right]^{2} \frac{2 z \cos \phi}{\Delta} F_{z} \\
& \approx-\frac{\delta^{2} s \sqrt{2 s} \cos \phi}{\Delta} F_{z} \\
& \approx-\frac{\delta^{2} s \sqrt{2} \cos \phi}{\Omega} F_{z} .
\end{aligned}
$$

Once again, if the energy gap of $H^{(1)}$ [Eq. (B19)] is larger than this perpendicular term, and assuming $\sqrt{2} \cos \phi \delta \ll \Omega$, we get only a small, third-order correction

$$
H^{(2)} \approx 2 \frac{s \delta^{3} \cos ^{2} \phi}{\Omega^{2}} F_{x}
$$

We find for both $s \gg 1$ and $s=1$ that the double-dressing scheme eliminates the first- and second-order contributions of the inhomogeneous shift. Note that when $s \neq 1$ but not large and $\phi \neq \pi / 2$, there will be a nonvanishing secondorder contribution.

To fully evaluate the contribution of the inhomogeneous shift to third order, we take the third order of the Magnus expansion

$$
H^{(3)}=-\frac{1}{6 t} \int_{0}^{t} d t_{1} \int_{0}^{t_{1}} d t_{2} \int_{0}^{t_{2}} d t_{3}\left(\left[H_{y}^{\prime}\left(t_{1}\right),\left[H_{y}^{\prime}\left(t_{2}\right), H_{y}^{\prime}\left(t_{3}\right)\right]\right]+\left[H_{y}^{\prime}\left(t_{3}\right),\left[H_{y}^{\prime}\left(t_{2}\right), H_{y}^{\prime}\left(t_{1}\right)\right]\right]\right) .
$$

For a general $s$, we obtain

$$
\begin{aligned}
H^{(3)}= & {\left[\frac{\delta(s+1)}{2}\right]^{3} \sum_{n>0}\left\{\frac{4\left(J_{2 n}(z)\right)^{3}}{(2 n \Delta)^{2}} \cos (2 n \phi) F_{x}-\frac{4\left(J_{2 n-1}(z)\right)^{3}}{((2 n-1) \Delta)^{2}} \sin ((2 n-1) \phi) F_{y}\right\} } \\
& -\left[\frac{\delta(s+1)}{2}\right]^{3} J_{0}(z) \sum_{n>0} \frac{2(2+\cos (2 n \phi))\left(J_{n}(z)\right)^{2}}{(n \Delta)^{2}} F_{x} \\
& +\left[\frac{\delta(s+1)}{2}\right]^{3}\left(J_{0}(z)\right)^{2} \sum_{n>0} \frac{8 J_{2 n-1}(z)}{((2 n-1) \Delta)^{2}} \sin ((2 n-1) \phi) F_{y} \\
& -\left[\frac{\delta(s+1)}{2}\right]^{3} J_{0}(z) \sum_{n \neq m \neq 0} \frac{4 J_{n}(z) J_{m}(z)}{\Delta^{2}}\left\{\frac{\cos n \phi \cos (m \phi)}{n m} F_{x}+\frac{m \cos (n \phi) \sin (m \phi)-n \sin (n \phi) \cos (m \phi)}{n m(m-n)} F_{y}\right\} .
\end{aligned}
$$

For $s=1$, this becomes

$$
H^{(3)}=\delta^{3} \sum_{n>0}\left\{\frac{4\left(J_{2 n}(z)\right)^{3}}{(2 n \Delta)^{2}} \cos (2 n \phi) F_{x}-\frac{4\left(J_{2 n-1}(z)\right)^{3}}{((2 n-1) \Delta)^{2}} \sin ((2 n-1) \phi) F_{y}\right\} \approx \frac{\delta^{3}}{\Omega^{2}}\left(0.06 \cos (2 \phi) F_{x}-0.4 \sin (\phi) F_{y}\right) .
$$

Here, we have used the fact that around the protection condition, the sum is well approximated by the first term. Since for $s=1$ the energy gap from Eq. (B19) is vanishing, both terms $\left(F_{x}\right.$ and $\left.F_{y}\right)$ contribute to the noise. The correction to the transition frequency due to the inhomogeneous shift thus varies in the range

$$
\Delta \omega_{\delta} \in(0.06,0.4) \times \frac{\delta^{3}}{\Omega^{2}},
$$

depending on the phase $\phi$. The lower noise amplitude is obtained when the two tones of the dressing are in phase, $\phi=0$.
For $s \gg 1$, the third-order Magnus term becomes

$H^{(3)} \approx \frac{\delta^{3}}{\Omega^{2}}\left[-s(2+\cos 2 \phi) F_{x}+\sqrt{2} \sin \phi s^{3 / 2} F_{y}\right]$,

which should be added to the second-order Magnus term [Eq. (B25)]

$$
H_{\mathrm{eff}}^{(2)}+H^{(3)} \approx \frac{\delta^{3}}{\Omega^{2}}\left(-s F_{x}+\sqrt{2} \sin \phi s^{3 / 2} F_{y}\right) .
$$

Once again, due to the large energy gap in the $F_{x}$ direction, the perpendicular term $\left(\propto F_{y}\right)$ produces a negligible 
perturbation. Therefore, when $s \gg 1$, the first nonvanishing order correction to the transition frequency due to the inhomogeneous shift is

$$
\Delta \omega_{\delta} \approx \frac{s \delta^{3}}{\Omega^{2}} .
$$

A summary of these leading terms is presented in Table II (middle section). The decay of coherence due to this thirdorder correction is non-Gaussian, due to the nonlinear coupling to shift $\delta$, and can be found by taking the Fourier transform of the transition frequency spectrum.

\section{Protection by a dressing field with a stepwise-modulated phase}

In our experiment, we did not explicitly employ the twotone (symmetrically detuned) dressing of Eq. (B7). Instead, we passed a single-tone, resonant $(\Delta=0)$ field through an electro-optic modulator and alternated its phase stepwise between 0 and $\pi$ with a half-period $T / 2$, which forms a multitone signal with a vanishing carrier, dominant firstorder side bands, and additional higher-order side bands. The power spectral density of this signal thus highly resembles the pure two-tone case discussed in the previous section. However, an exact solution may be obtained for this case. The Hamiltonian alternates between $H_{+}$and $H_{-}$, where $H_{ \pm}=H_{0} \pm \Omega \sigma_{x}$. In analogy to the phase $\phi$ of the two-tone dressing in the previous section, here we control the duration of the initial interval (relative to the qubit initialization time $t=0$ ). We can describe the unitary evolution over the first full period $T$ with

$$
U_{T}=e^{-i H_{-}[1-f(\phi)] T / 2} e^{-i H_{+} T / 2} e^{-i H_{-} f(\phi) T / 2},
$$

where $f(\phi)=\cos ^{2}(\pi / 4-\phi / 2)$. For an evolution time $t=n T$, the evolution operator is $U(t)=\left(U_{T}\right)^{n}$. One can see that the stepwise dressing with period $T$ corresponds to the two-tone dressing with detuning $\Delta=2 \pi / T$. To expand $U_{T}$ in leading orders of the inhomogeneous shift $\delta$ and the drive noise $\delta_{\Omega}$, we define an effective Hamiltonian $H_{\text {eff }}$ by $U_{T}=\exp \left(-i H_{\text {eff }} 2 \pi / \Delta\right)$, such that $H_{\text {eff }}$ can be expanded in orders of $\delta$. The first order reads

$$
\begin{aligned}
H^{(1)}= & \delta \frac{s-1}{2} \mathbb{I}-\delta \frac{s+1}{2} \frac{\sin (\pi \Omega / \Delta)}{\pi \Omega / \Delta}\left[\cos [(\pi \Omega / \Delta) \sin \phi] \sigma_{z}\right. \\
& \left.-\sin [(\pi \Omega / \Delta) \sin \phi] \sigma_{y}\right] .
\end{aligned}
$$

Therefore, for protection up to first order we require

$$
\sin c(\pi \Omega / \Delta)=\frac{s-1}{s+1},
$$

where $\sin c(x)=\sin (x) / x$. The protected dressed state is the eigenstate of the operator $F_{x}=-\cos [(\pi \Omega / \Delta) \sin \phi] \sigma_{z}-$ $\sin [(\pi \Omega / \Delta) \sin \phi] \sigma_{y}$ with the eigenvalue -1 [analogous to Eq. (B19)]. For $s \gg 1$, the condition (B35) can be expanded to leading order, $1-\frac{(\pi \Omega / \Delta)^{2}}{6}+O\left((\pi \Omega / \Delta)^{4}\right)=1-\frac{2}{s}+O\left(s^{-2}\right)$.

We thus arrive at the condition $(\Omega / \Delta)^{2}=12 / \pi^{2} s \approx 1 / s$, in agreement with both the one-tone and two-tone dressings at the $s \gg 1$ limit.

\section{a. Higher-order noise contributions}

We maintain the notations of parallel $\left(\propto F_{x}\right)$ and perpendicular $\left(\propto F_{y}, F_{z}\right)$ noise terms in the dressedstate basis, where now $F_{y}=\cos [(\pi \Omega / \Delta) \sin \phi] \sigma_{y}-$ $\sin [(\pi \Omega / \Delta) \sin \phi] \sigma_{z}$ and $F_{z}=\sigma_{x}$. The cross term of the drive noise, together with the inhomogeneous shift, is

$$
\begin{aligned}
H_{\delta \cdot \delta_{\Omega}}^{(1)}= & \frac{1}{\Omega}\left\{[\cos (\pi \Omega / \Delta)-\sin c(\pi \Omega / \Delta)] F_{x}\right. \\
& \left.-\sin \phi \sin (\pi \Omega / \Delta) F_{y}\right\}\left[\frac{s+1}{2} \delta \delta_{\Omega}\right] .
\end{aligned}
$$

As in the two-tone case, the perpendicular contribution vanishes when $\phi \rightarrow 0$. For a general $\phi$, we examine the limits $s=1$ and $s \gg 1$ :

(i) For $s=1$, under the protection condition (B35), the perpendicular term vanishes, and we are left with the parallel term

$$
\Delta \omega_{\delta \cdot \delta_{\Omega}}=\frac{\delta \delta_{\Omega}}{\Omega} .
$$

(ii) For $s \gg 1$, this perpendicular term gives rise to a higher-order perturbation when the energy gap $\delta(s+1)$ [Eq. (B34)] is large enough. The leading contribution in the parallel term is

$$
\Delta \omega_{\delta \cdot \delta_{\Omega}}=2 \frac{\delta \delta_{\Omega}}{\Omega} .
$$

To second order in $\delta$, the effective Hamiltonian reads

$$
\begin{aligned}
H^{(2)}= & \frac{1}{\Omega} \sin c(\pi \Omega / \Delta)(\cos \pi \Omega / \Delta-\cos [(\pi \Omega / \Delta) \sin \phi]) \\
& \times\left(\frac{s+1}{2} \delta\right)^{2} F_{z} .
\end{aligned}
$$

As in the two-tone case, the second-order contribution vanishes for $\phi \rightarrow \pi / 2$ or for $s=1$. For $s \gg 1$, we are left with $H^{(2)} \approx-\cos ^{2} \phi(3 / 2)\left(\delta^{2} / \Omega\right) F_{z}$, which is perpendicular to the dressed-state basis and thus only contributes a third-order correction

$$
H^{(2)} \approx \cos ^{4} \phi \frac{9 s}{4 \Omega^{2}} \delta^{3} F_{x},
$$

assuming a large enough energy gap $\delta(s+1)$. For general $\phi$ and $s$, the second-order term does not vanish. The 
effective Hamiltonian to the third order, for $s=1$, is $H^{(3)}=-\delta^{3} /\left(2 \Omega^{2}\right) F_{x}$. For $s \gg 1$, the third-order term is

$$
H^{(3)}=-3 s \frac{64+120 \cos ^{4} \phi}{160 \Omega^{2}} \delta^{3} F_{x},
$$

and, together with the higher-order contribution of $H^{(2)}$, we obtain $H^{(2)}+H^{(3)} \approx-\left(6 s / 5 \Omega^{2}\right) \delta^{3} F_{x}$. The first nonvanishing order correction to the transition frequency due to the inhomogeneous shift is thus

$$
\Delta \omega_{\delta}=\frac{6 s}{5 \Omega^{2}} \delta^{3} .
$$

A summary of these leading terms is presented in Table II (right section).

[1] S. Lepoutre, K. Kechadi, B. Naylor, B. Zhu, L. Gabardos, L. Isaev, P. Pedri, A. M. Rey, L. Vernac, and B. Laburthe-Tolra, Spin Mixing and Protection of Ferromagnetism in a Spinor Dipolar Condensate, Phys. Rev. A 97, 023610 (2018).

[2] M. Munowitz and A. Pines, Multiple-Quantum Nuclear Magnetic Resonance Spectroscopy, Science 233, 525 (1986).

[3] S. Kotler, N. Akerman, Y. Glickman, A. Keselman, and R. Ozeri, Single-Ion Quantum Lock-in Amplifier, Nature (London) 473, 61 (2011).

[4] B. Pingault, J. N. Becker, C. H. H. Schulte, C. Arend, C. Hepp, T. Godde, A. I. Tartakovskii, M. Markham, C. Becher, and M. Atatüre, All-Optical Formation of Coherent Dark States of Silicon-Vacancy Spins in Diamond, Phys. Rev. Lett. 113, 263601 (2014).

[5] D. Cogan, O. Kenneth, N. H. Lindner, G. Peniakov, C. Hopfmann, D. Dalacu, P. J. Poole, P. Hawrylak, and D. Gershoni, Depolarization of Electronic Spin Qubits Confined in Semiconductor Quantum Dots, Phys. Rev. X 8, 041050 (2018).

[6] R. Finkelstein, O. Lahad, O. Michel, O. Davidson, E. Poem, and O. Firstenberg, Power Narrowing: Counteracting Doppler Broadening in Two-Color Transitions, New J. Phys. 21, 103024 (2019).

[7] R. Finkelstein, E. Poem, O. Michel, O. Lahad, and O. Firstenberg, Fast, Noise-Free Memory for Photon Synchronization at Room Temperature, Sci. Adv. 4, eaap8598 (2018).

[8] A. Kaplan, M. F. Andersen, and N. Davidson, Suppression of Inhomogeneous Broadening in rf Spectroscopy of Optically Trapped Atoms, Phys. Rev. A 66, 045401 (2002).

[9] H. Levine, A. Keesling, A. Omran, H. Bernien, S. Schwartz, A. S. Zibrov, M. Endres, M. Greiner, V. Vuletić, and M. D. Lukin, High-Fidelity Control and Entanglement of RydbergAtom Qubits, Phys. Rev. Lett. 121, 123603 (2018).

[10] M. Saffman, Quantum Computing with Atomic Qubits and Rydberg Interactions: Progress and Challenges, J. Phys. B 49, 202001 (2016).
[11] P. Senellart, G. Solomon, and A. White, High-Performance Semiconductor Quantum-Dot Single-Photon Sources, Nat. Nanotechol. 11, 1026 (2017).

[12] P. Krantz, M. Kjaergaard, F. Yan, T. P. Orlando, S. Gustavsson, and W.D. Oliver, A Quantum Engineer's Guide to Superconducting Qubits, Appl. Phys. Rev. 6, 021318 (2019).

[13] M. H. Devoret and R. J. Schoelkopf, Superconducting Circuits for Quantum Information: An Outlook, Science 339, 1169 (2013).

[14] T. Manovitz, R. Shaniv, Y. Shapira, R. Ozeri, and N. Akerman, Precision Measurement of Atomic Isotope Shifts Using a Two-Isotope Entangled State, Phys. Rev. Lett. 123, 203001 (2019).

[15] N. Aharon, M. Drewsen, and A. Retzker, General Scheme for the Construction of a Protected Qubit Subspace, Phys. Rev. Lett. 111, 230507 (2013).

[16] A. Bermudez, P. O. Schmidt, M. B. Plenio, and A. Retzker, Robust Trapped-Ion Quantum Logic Gates by Continuous Dynamical Decoupling, Phys. Rev. A 85, 040302(R) (2012).

[17] D. M. Harber, H. J. Lewandowski, J. M. McGuirk, and E. A. Cornell, Effect of Cold Collisions on Spin Coherence and Resonance Shifts in a Magnetically Trapped Ultracold Gas, Phys. Rev. A 66, 053616 (2002).

[18] J. Ye, H. J. Kimble, and H. Katori, Quantum State Engineering and Precision Metrology Using State-Insensitive Light Traps, Science 320, 1734 (2008).

[19] A. G. Radnaev, Y. O. Dudin, R. Zhao, H. H. Jen, S. D. Jenkins, A. Kuzmich, and T. A. Kennedy, A Quantum Memory with Telecom-Wavelength Conversion, Nat. Phys. 6, 894 (2010).

[20] O. Lahad, R. Finkelstein, O. Davidson, O. Michel, E. Poem, and O. Firstenberg, Recovering the Homogeneous Absorption of Inhomogeneous Media, Phys. Rev. Lett. 123, 173203 (2019).

[21] A. P. Hilton, C. Perrella, A. N. Luiten, and P. S. Light, DualColor Magic-Wavelength Trap for Suppression of Light Shifts in Atoms, Phys. Rev. Applied 11, 024065 (2019).

[22] L. Viola, E. Knill, and S. Lloyd, Dynamical Decoupling of Open Quantum Systems, Phys. Rev. Lett. 82, 2417 (1999).

[23] K. Khodjasteh and D. A. Lidar, Fault-Tolerant Quantum Dynamical Decoupling, Phys. Rev. Lett. 95, 180501 (2005).

[24] A. M. Souza, G. A. Álvarez, and D. Suter, Robust Dynamical Decoupling for Quantum Computing and Quantum Memory, Phys. Rev. Lett. 106, 240501 (2011).

[25] J. Rui, Y. Jiang, S. J. Yang, B. Zhao, X. H. Bao, and J. W. Pan, Operating Spin Echo in the Quantum Regime for an Atomic-Ensemble Quantum Memory, Phys. Rev. Lett. 115, 133002 (2015).

[26] F. F. Fanchini, J. E. M. Hornos, and R. d. J. Napolitano, Continuously Decoupling Single-Qubit Operations from a Perturbing Thermal Bath of Scalar Bosons, Phys. Rev. A 75, 022329 (2007).

[27] T. R. Tan, J. P. Gaebler, R. Bowler, Y. Lin, J. D. Jost, D. Leibfried, and D. J. Wineland, Demonstration of a DressedState Phase Gate for Trapped Ions, Phys. Rev. Lett. 110, 263002 (2013). 
[28] D. A. Golter, T. K. Baldwin, and H. Wang, Protecting a Solid-State Spin from Decoherence Using Dressed Spin States, Phys. Rev. Lett. 113, 237601 (2014).

[29] R. Zhao, Y. O. Dudin, S. D. Jenkins, C. J. Campbell, D. N. Matsukevich, T. A. Kennedy, and A. Kuzmich, Long-Lived Quantum Memory, Nat. Phys. 5, 100 (2009).

[30] Y. O. Dudin, L. Li, and A. Kuzmich, Light Storage on the Time Scale of a Minute, Phys. Rev. A 87, 031801(R) (2013).

[31] S. J. Yang, X. J. Wang, X. H. Bao, and J. W. Pan, An Efficient Quantum Light-Matter Interface with Sub-second Lifetime, Nat. Photonics 10, 381 (2016).

[32] M. Körber, O. Morin, S. Langenfeld, A. Neuzner, S. Ritter, and G. Rempe, Decoherence-Protected Memory for a Single-Photon Qubit, Nat. Photonics 12, 18 (2018).

[33] O. Katz and O. Firstenberg, Light Storage for One Second in Room-Temperature Alkali Vapor, Nat. Commun. 9, 2074 (2018).

[34] K. T. Kaczmarek, P. M. Ledingham, B. Brecht, S. E. Thomas, G. S. Thekkadath, O. Lazo-Arjona, J. H. D. Munns, E. Poem, A. Feizpour, D. J. Saunders, J. Nunn, and I. A. Walmsley, High-Speed Noise-Free Optical Quantum Memory, Phys. Rev. A 97, 042316 (2018).

[35] B. Julsgaard, A. Kozhekin, and E. S. Polzik, Experimental Long-Lived Entanglement of Two Macroscopic Objects, Nature (London) 413, 400 (2001).

[36] H. Krauter, D. Salart, C. A. Muschik, J. M. Petersen, H. Shen, T. Fernholz, and E. S. Polzik, Deterministic Quantum Teleportation between Distant Atomic Objects, Nat. Phys. 9 , 400 (2013).

[37] M. Lettner, M. Mücke, S. Riedl, C. Vo, C. Hahn, S. Baur, J. Bochmann, S. Ritter, S. Dürr, and G. Rempe, Remote Entanglement between a Single Atom and a Bose-Einstein Condensate, Phys. Rev. Lett. 106, 210503 (2011).

[38] L. M. Duan, M. D. Lukin, J. I. Cirac, and P. Zoller, LongDistance Quantum Communication with Atomic Ensembles and Linear Optics, Nature (London) 414, 413 (2001).

[39] V. Boyer, A. M. Marino, R. C. Pooser, and P. D. Lett, Entangled Images from Four-Wave Mixing, Science 321, 544 (2008).

[40] D. J. Whiting, N. Šibalić, J. Keaveney, C. S. Adams, and I. G. Hughes, Single-Photon Interference Due to Motion in an Atomic Collective Excitation, Phys. Rev. Lett. 118, 253601 (2017).

[41] S. A. Moiseev and S. Kröll, Complete Reconstruction of the Quantum State of a Single-Photon Wave Packet Absorbed by a Doppler-Broadened Transition, Phys. Rev. Lett. 87, 173601 (2001).

[42] A. Stark, N. Aharon, A. Huck, H. A. El-Ella, A. Retzker, F. Jelezko, and U. L. Andersen, Clock Transition by Continuous Dynamical Decoupling of a Three-Level System, Sci. Rep. 8, 14807 (2018).

[43] D. Trypogeorgos, A. Valdés-Curiel, N. Lundblad, and I. B. Spielman, Synthetic Clock Transitions via Continuous Dynamical Decoupling, Phys. Rev. A 97, 013407 (2018).

[44] D. F. Phillips, A. Fleischhauer, A. Mair, R. L. Walsworth, and M. D. Lukin, Storage of Light in Atomic Vapor, Phys. Rev. Lett. 86, 783 (2001).

[45] See Supplemental Material at http://link.aps.org/ supplemental/10.1103/PhysRevX.11.011008 for time evolution of a collective excitation in a thermal atomic ensemble without a dressing field, with a single dressing field, and with a double dressing field in Video 1.

[46] S. Schmidt-Eberle, T. Stolz, G. Rempe, and S. Dürr, DarkTime Decay of the Retrieval Efficiency of Light Stored as a Rydberg Excitation in a Noninteracting Ultracold Gas, Phys. Rev. A 101, 013421 (2020).

[47] Y. Sung, F. Beaudoin, L. M. Norris, F. Yan, D. K. Kim, J. Y. Qiu, U. von Lüpke, J. L. Yoder, T. P. Orlando, S. Gustavsson, L. Viola, and W. D. Oliver, Non-Gaussian Noise Spectroscopy with a Superconducting Qubit Sensor, Nat. Commun. 10, 1 (2019).

[48] M. Parniak, M. Mazelanik, A. Leszczynski, M. Lipka, M. Dabrowski, and W. Wasilewski, Quantum Optics of Spin Waves through ac Stark Modulation, Phys. Rev. Lett. 122, 063604 (2019).

[49] T. M. Graham, M. Kwon, B. Grinkemeyer, Z. Marra, X. Jiang, M. T. Lichtman, Y. Sun, M. Ebert, and M. Saffman, Rydberg-Mediated Entanglement in a Two-Dimensional Neutral Atom Qubit Array, Phys. Rev. Lett. 123, 230501 (2019).

[50] F. Ripka, H. Kübler, R. Löw, and T. Pfau, A RoomTemperature Single-Photon Source Based on Strongly Interacting Rydberg Atoms, Science 362, 446 (2018).

[51] Y.-S. Lee, S. M. Lee, H. Kim, and H. S. Moon, Highly Bright Photon-Pair Generation in Doppler-Broadened Ladder-Type Atomic System, Opt. Express 24, 28083 (2016).

[52] A. Stark, N. Aharon, T. Unden, D. Louzon, A. Huck, A. Retzker, U. L. Andersen, and F. Jelezko, NarrowBandwidth Sensing of High-Frequency Fields with Continuous Dynamical Decoupling, Nat. Commun. 8, 1105 (2017).

[53] R. T. Sutherland, R. Srinivas, S. C. Burd, D. Leibfried, A. C. Wilson, D. J. Wineland, D. T. Allcock, D. H. Slichter, and S. B. Libby, Versatile Laser-Free Trapped-Ion Entangling Gates, New J. Phys. 21, 033033 (2019).

[54] Consider three states $\left|\psi_{n}\right\rangle(n=1,2,3)$ with energies $\hbar \omega_{n}\left(\delta^{*}\right)=\hbar\left(\omega_{n}^{0}+a_{n} \delta^{*}\right)$, arranged such that $a_{1} \leq a_{2} \leq a_{3}$. If $a_{1}=a_{2}$ or $a_{2}=a_{3}$, then the corresponding transition is already protected from dephasing due to changes in $\delta^{*}$. Otherwise, denote $n_{ \pm}=2 \pm \operatorname{sign}\left(a_{1}-2 a_{2}+a_{3}\right)$ and define $|\downarrow\rangle \equiv\left|\psi_{2}\right\rangle,|\uparrow\rangle \equiv\left|\psi_{n_{-}}\right\rangle,|S\rangle \equiv\left|\psi_{n_{+}}\right\rangle, \delta \equiv\left(a_{2}-a_{n_{-}}\right) \delta^{*}$, and $s \equiv\left(a_{n_{+}}-a_{2}\right) /\left(a_{2}-a_{n_{-}}\right)$.

[55] P. Facchi, D. A. Lidar, and S. Pascazio, Unification of Dynamical Decoupling and the Quantum Zeno Effect, Phys. Rev. A 69, 032314 (2004).

[56] D. F. James and J. Jerke, Effective Hamiltonian Theory and Its Applications in Quantum Information, Can. J. Phys. 85, 625 (2007).

[57] R. Srinivas, S. C. Burd, R. T. Sutherland, A. C. Wilson, D. J. Wineland, D. Leibfried, D. T. Allcock, and D. H. Slichter, Trapped-Ion Spin-Motion Coupling with Microwaves and a Near-Motional Oscillating Magnetic Field Gradient, Phys. Rev. Lett. 122, 163201 (2019).

[58] C.F. Roos, Ion Trap Quantum Gates with AmplitudeModulated Laser Beams, New J. Phys. 10, 013002 (2008). 